\title{
SPECTRAL PROPERTIES OF CLASSICAL WAVES IN HIGH-CONTRAST PERIODIC MEDIA*
}

\author{
A. FIGOTIN $^{\dagger}$ AND P. KUCHMENT ${ }^{\ddagger}$
}

\begin{abstract}
We introduce and investigate the band gap structure of the frequency spectrum for classical electromagnetic and acoustic waves in a high-contrast, two-component periodic medium. The asymptotics with respect to the high-contrast is considered. The limit medium is described in terms of appropriate self-adjoint operators and the convergence to the limit is proven. These limit operators give an idea of the spectral structure and suggest new numerical approaches as well. The results are obtained in arbitrary dimension and for rather general geometry of the medium. In particular, two-dimensional (2D) photonic band gap structures and their acoustic analogues are covered.
\end{abstract}

Key words. propagation of electromagnetic and acoustic waves, band gap structure of the spectrum, periodic dielectrics, periodic acoustic media, high contrast

AMS subject classifications. 35B27, 73D25, 78A45

PII. S0036139996297249

1. Introduction. Wave propagation in inhomogeneous media is qualitatively different than in homogeneous ones. The reason for this is the coherent multiple scattering and interference. In periodic media the multiple scattering can manifest itself in the rise of the so-called stop bands or gaps in the frequency spectrum. If the wave frequency falls in a gap, then such a wave cannot propagate in the medium. In disordered media, coherent multiple scattering and interference can result in existence of exponentially localized eigenmodes (the phenomenon known as Anderson localization). The transport of classical electromagnetic, acoustic, and elastic waves in periodic and disordered media has recently been studied very intensively, both theoretically and experimentally (see [12], [13], [20], [1], [11], [16], [21]). In particular, artificial periodic structures have been studied with the goal of finding conditions that favor band gaps in the spectrum. Fabrication of materials with prescribed spectral gaps could lead to exciting opportunities in manufacturing entirely new and/or improved devices (see [1], [13], [21], and references therein).

This paper is a continuation of the series of papers [4], [5], [6], [7], [8], [9], whose goal is to develop some mathematical tools that can provide qualitative and quantitative understanding of the dependence of spectral properties (such as band gap structure, eigenmodes, etc.) on the parameters of the periodic medium. High-contrast media, due to their strong scattering properties, constitute a natural and important subject of theoretical investigation. Let us describe the definition of the high-contrast

\footnotetext{
* Received by the editors January 17, 1996; accepted for publication (in revised form) November 2, 1996. The U.S. Government is authorized to reproduce and distribute reprints for governmental purposes notwithstanding any copyright notation thereon. The views and conclusions contained herein are those of the authors and should not be interpreted as necessarily representing the official policies or endorsements, either expressed or implied, of the Air Force Office of Scientific Research, the National Science Foundation, or the U.S. Government.

http://www.siam.org/journals/siap/58-2/29724.html

$\dagger$ Mathematics Department, University of North Carolina, Charlotte, NC 28223 (figotin@ mosaic.uncc.edu). The research of this author was supported by U.S. Air Force, Air Force Office of Scientific Research, and Air Force Material Command grant F49620-94-1-0172DEF.

$\ddagger$ Department of Mathematics and Statistics, Wichita State University, Wichita, KS 67260-0033 (kuchment@twsuvn.uc.twsu.edu). The research of this author was partially supported by Division of Mathematical Sciences of the NSF grant DMS 910211 and by an NSF EPSCOR grant.
} 

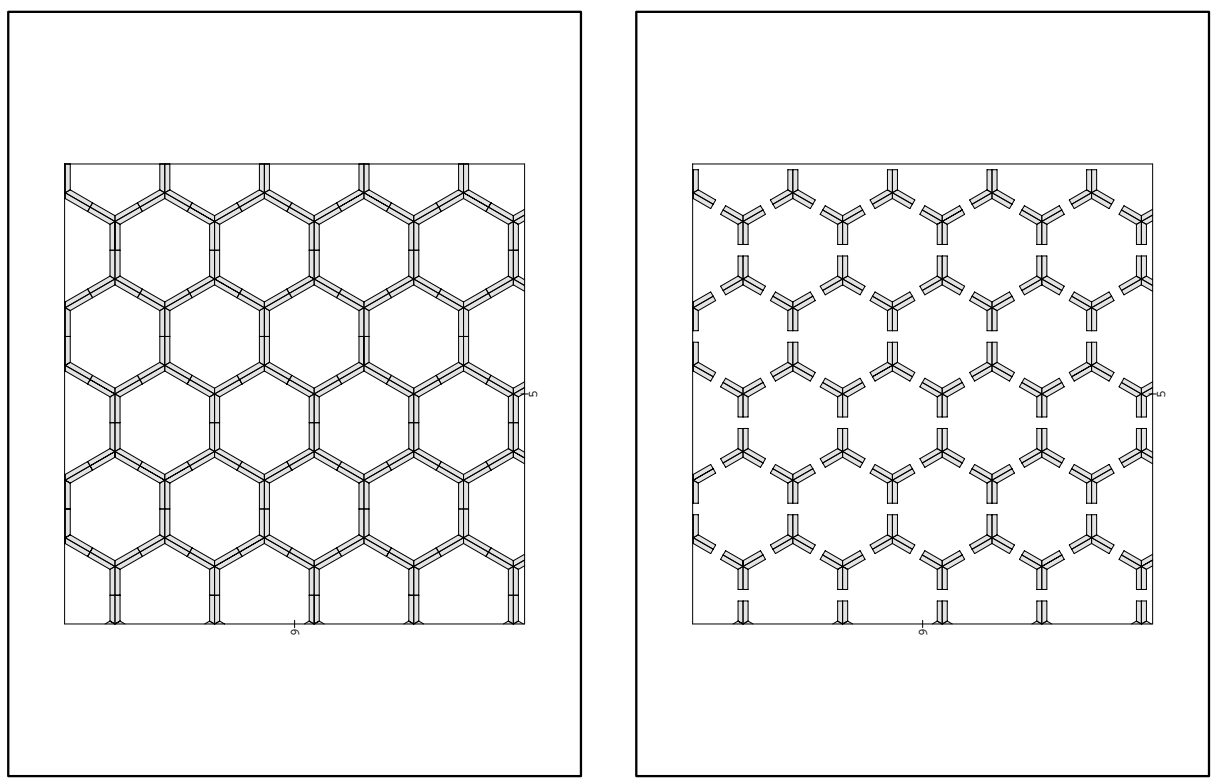

FIG. 1. The figure on the left shows a periodic pattern when the light component of the medium is disconnected. The figure on the right shows a periodic pattern when the light component is connected.

media adopted in this paper. Suppose that spacial properties of the medium are described by a scalar position-dependent function $\varepsilon(\mathbf{x})$ (which is the dielectric constant for dielectric media, or the compressibility for acoustic media). Suppose also that the medium consists of two components and correspondingly the function $\varepsilon(\mathbf{x})$ takes on just two values, say 1 and $\epsilon>1$. Let us call the component of the medium where $\varepsilon(\mathbf{x})=1$ "light" and the second one where $\varepsilon(\mathbf{x})=\epsilon$ "dense." We define a highcontrast medium as one having the following properties: (i) the dense component is a set of thin slabs of the thickness $\alpha \ll 1$, so its volume fraction is of order $\alpha \ll 1$; (ii) the total "mass" of the dense component per unit volume $\epsilon \alpha$ does not approach zero (in particular, it can be very large).

An important example of high-contrast periodic media is the one where the dense component forms a connected set, while the light component forms a disconnected one (see Fig. 1). In this case we can view the medium as a periodic array of "air bubbles" separated by a thin, dense film of the thickness $\alpha$. The case of such $2 \mathrm{D}$ periodic dielectric or acoustic media, when the domains are columns of square cross-section and the periods form a simple cubic lattice, was studied in our papers [6], [7], [8], [9] under the conditions that

$$
\epsilon \alpha \gg 1, \epsilon \alpha^{2} \ll 1 \text {. }
$$

Along with the detailed analysis of the band gap structure (in particular, existence of absolute gaps), we also discovered two types of eigenmodes that correspond to two qualitatively different parts of the frequency spectrum. The eigenmodes of the first type are well confined to the thin, dense component. The corresponding part of the spectrum consists of small bands of order $(\epsilon \alpha)^{-1}$, which alternate with small gaps of the same order. The structure of those eigenmodes suggests that they presumably arise due to the phenomenon of total internal reflection, which is well known in optics. In that case the thin film of the dense material plays the role of a wave guide for these 
eigenmodes. There also is another part of the spectrum that corresponds to waves whose energy resides primarily in the light component. This part of the spectrum asymptotically (i.e., under conditions in (1)) tends to be almost discrete with large gaps between narrow spectral bands of order $(\epsilon \alpha)^{-1}$ (see [6], [7], and [8] for details). Similar results also should hold for two-component media of rather arbitrary complicated geometries as long as the conditions (1) are satisfied. The natural idea is that for the high-contrast media (in particular, under the limit conditions (1)) the operator governing the spectrum asymptotically splits into two operators that are responsible for two different parts of the spectrum. One can expect from the results of [6], [7], [8], [9] that the operator governing the waves that are concentrated in the "light" component is either Dirichlet or Neumann (depending on polarization) Laplacian in the single cell of the "light" material. It is not immediately clear, however, what operator is responsible for the waves propagating along the thin, dense component. It is even less clear how the asymptotic splitting occurs. Another problem is that the technique used in [6], [7], [8], [9] is based on separation of variables and therefore is not applicable to geometries more complicated than the cubic one.

In this paper we do the following:

1. Find a high-contrast limit operator (which in some interesting cases can be represented as a version of the so-called Dirichlet-to-Neumann map) that is responsible for the spectrum of waves residing in the thin film of dense component.

2. Give a rigorous proof that this operator is the true limit of the operators with finite $\epsilon$ and $\alpha$ and that it gives the correct approximation for the spectrum in the case of high contrast.

3. Provide an approach that works in arbitrary dimension and for general geometry.

4. Reduce (1) to much less restrictive asymptotic conditions.

The results of the paper also suggest some new numerical approaches to the analysis of photonic band gap structures and of their acoustic counterparts. The numerical implementation, as well as analysis of the complete Maxwell system, will be discussed elsewhere.

Now we will introduce the mathematical framework of the problem and formulate the main results.

Let us consider first the case when the dense component is connected and forms a thin film of the thickness $\alpha$ (see Fig. 1). Consider the Euclidean space $\mathbb{R}^{d}$. Suppose that the space is tiled periodically by polyhedra (polygons in the $2 \mathrm{D}$ case) $\Omega_{p}$ without overlapping: $\mathbb{R}^{d}=\bigcup_{p} \Omega_{p}$. We also assume that this tiling is invariant with respect to some lattice $\Gamma$ (discrete group of translations with a compact fundamental domain) in $\mathbb{R}^{d}$. The simplest example is the cubic structure, when $\Gamma$ is the integer lattice

$$
\Gamma=\mathbb{Z}^{d}=\left\{\mathbf{n}=\left(n_{1}, \ldots, n_{d}\right) \mid n_{j} \in \mathbb{Z}, j=1, \ldots, d\right\},
$$

the fundamental polyhedron is

$$
\Omega_{0}=\left\{x=\left(x_{1}, \ldots, x_{d}\right) \mid 0 \leq x_{j} \leq 1, j=1, \ldots, d\right\},
$$

and all other polyhedra are obtained by $\Gamma$ translations of $\Omega_{0}$ :

$$
\Omega_{p}=p+\Omega_{0}, p \in \Gamma
$$


Let $\partial \Omega$ denote the boundary of the domain $\Omega$. We introduce the following surface:

$$
\Sigma=\bigcup_{p} \partial \Omega_{p}
$$

(in the 2D case this is just a periodic graph in the plane). This surface (graph) plays the central role in our consideration.

Given a small parameter $\alpha<1$, we construct the $\alpha$-neighborhood $\Sigma^{\alpha}$ of $\Sigma$, namely

$$
\Sigma^{\alpha}=\left\{x \in \mathbb{R}^{d} \mid \operatorname{dist}(x, \Sigma) \leq \alpha\right\} .
$$

Then we define smaller domains $\Omega_{p}^{\alpha}$ as

$$
\Omega_{p}^{\alpha}=\Omega_{p} \backslash \Sigma^{\alpha}, \quad \Omega^{\alpha}=\bigcup_{p} \Omega_{p}^{\alpha},
$$

and

$$
\Sigma^{\alpha}=\mathbb{R}^{d} \backslash \Omega^{\alpha}, \quad \mathbb{R}^{d}=\Sigma^{\alpha} \bigcup \Omega^{\alpha} .
$$

In fact, this particular construction of the $\alpha$-neighborhood $\Sigma^{\alpha}$ is not very important. For instance, if $\Omega_{p}$ is convex, then we can choose a point $z_{p}$ in each of the domains $\Omega_{p}$ in such a way that this choice is invariant under the group $\Gamma$ and introduce $\Omega_{p}^{\alpha}$ as the result of the dilation with the factor $(1-\alpha / 2)$ and the center $z_{p}$ applied to $\Omega_{p}$.

The general case, when the dense component of the medium is not connected (see Fig. 1), can be described by a simple modification of the construction for the connected case. Namely, instead of $\Sigma$ we just take some of its periodic polyhedral subset $\breve{\Sigma} \subset \Sigma$. Then we deal with the sets $\breve{\Sigma}, \breve{\Sigma}^{\alpha}$, and $\breve{\Omega}_{p}^{\alpha}=\Omega_{p} \backslash \breve{\Sigma}^{\alpha}$ instead of $\Sigma, \Sigma^{\alpha}$, and $\Omega_{p}^{\alpha}$, and most of the constructions do not change.

Suppose now that two parts of the space $\mathbb{R}^{d}=\Sigma^{\alpha} \bigcup \Omega^{\alpha}$ are filled with two different materials. We assume that $\varepsilon(\mathbf{x})=1$ on $\Omega^{\alpha}$ (i.e., $\Omega^{\alpha}$ consists of the "air bubbles" $\Omega_{p}^{\alpha}$ ) and $\varepsilon(\mathbf{x})=\epsilon>>1$ on the rest of the medium. Hence, the coefficient describing our medium is defined by

$$
\varepsilon(\mathbf{x})=\left\{\begin{array}{lll}
1 & \text { if } & \mathbf{x} \in \Omega^{\alpha}, \\
\epsilon & \text { if } & \mathbf{x} \in \Sigma^{\alpha} .
\end{array}\right.
$$

Note that, due to the well-known scaling properties of the Maxwell system and of the equations for acoustic waves, the assumption that $\epsilon=1$ is not actually restrictive. We will also need the auxiliary parameter

$$
w=(\epsilon \alpha)^{-1} .
$$

We will refer to the function $\varepsilon(\mathbf{x})$ as to the dielectric function, although all considerations are applicable to the acoustic case as well. In both cases of electromagnetic and acoustic waves, the following spectral problem is important for describing propagation of monochromatic waves (see, for instance, [9], [17]):

$$
-\Delta \varphi(\mathbf{x})=\lambda \varepsilon(\mathbf{x}) \varphi(\mathbf{x}), \mathbf{x} \in \mathbb{R}^{d} .
$$

Here the spectral parameter represents the time frequency of the wave. For instance, in the electromagnetic case $\lambda=(\omega / c)^{2}$, where $\omega$ is the time frequency and $c$ is the speed of light. In the case of electromagnetic waves, however, this equation does not tell the whole story, since one needs here the complete Maxwell system. Furthermore, for $d=2$ this problem describes one possible wave polarization: electromagnetic 
waves polarized in such a way that the electric field $\mathbf{E}$ is normal to the plane of the material. (The second polarization when the electric field is orthogonal to the plane was considered in [6].) Anyway, in both acoustic and electromagnetic cases it is important to understand the structure of the spectrum of this problem (in particular, existence of spectral gaps). We will consider spectral properties of (3) under the conditions significantly weaker than (1).

The case of the 2D square structure (see above) was thoroughly studied in [7], [8], [9]. Let us assume now that $d=2, \Gamma=\mathbb{Z}^{2}$ (the integer lattice), and

$$
\Omega_{0}=\left\{x=\left(x_{1}, x_{2}\right) \mid 0 \leq x_{j} \leq 1, j=1,2\right\} .
$$

The result of [7], [8], [9] was that under the conditions in (1) any finite part of the spectrum splits into two subspectra with different asymptotic behavior. Namely, the following statement was proven.

THEOREM 1. The spectrum $\sigma$ of the problem (3) (in the case of square geometry in $\mathbb{R}^{2}$ ) splits into two subspectra

$$
\sigma=\sigma_{H} \cup \sigma_{E}
$$

that satisfy the following properties:

(i) For any finite interval $[0, N]$ and if the values $\epsilon \alpha^{4 / 3}$ and $w=(\epsilon \alpha)^{-1}$ are small enough, the intersection of the subspectrum $\sigma_{H}$ with the interval $[0, N]$ consists of the following union of intervals:

$$
\bigcup_{\mathbf{n} \in \mathbb{Z}_{+}^{2}, \mathbf{n} \neq 0}\left[(\pi \mathbf{n})^{2}-\rho_{\mathbf{n}}^{-},(\pi \mathbf{n})^{2}+\rho_{\mathbf{n}}^{+}\right] \bigcap[0, N] .
$$

Here, for the nonnegative integer vector $\mathbf{n}=\left(n_{1}, n_{2}\right) \in \mathbb{Z}_{+}^{2}$ we denote $\mathbf{n}^{2}=n_{1}^{2}+n_{2}^{2}$. The quantities $\rho_{\mathbf{n}}^{ \pm}$satisfy the estimate

$$
\left|\rho_{\mathbf{n}}^{ \pm}\right| \leq c w
$$

for all $\mathbf{n}$ such that the intersection in (4) is nonempty. All the intervals above contain nonempty portions of the spectrum.

In other words, any finite portion of the subspectrum $\sigma_{H}$ concentrates around the discrete set

$$
\bigcup_{\mathbf{n} \in \mathbb{Z}_{+}^{2}, \mathbf{n} \neq 0}\left\{(\pi \mathbf{n})^{2}\right\}
$$

which in fact is the Dirichlet spectrum for the Laplacian in the "air bubble" $\Omega_{0}$.

(ii) Under the same conditions as in (i), the intersection of the subspectrum $\sigma_{E}$ with the interval $[0, N]$ can be described as follows:

$$
\sigma_{E} \bigcap[0, N]=\left[\bigcup_{n \in \mathbb{Z}_{+}}\left[a_{n}, b_{n}\right]\right] \bigcap[0, N],
$$

where the intervals $\left[a_{n}, b_{n}\right]$ are disjoint and their lengths and gaps between them satisfy the estimates

$$
\begin{gathered}
c_{1} w \leq\left|a_{n}-b_{n}\right| \leq c_{2} w, \\
c_{1} w \leq\left|b_{n-1}-a_{n}\right| \leq c_{2} w .
\end{gathered}
$$


(iii) The Floquet-Bloch eigenmodes corresponding to the subspectrum $\sigma_{H}$ are localized mostly in the "air bubbles" $\Omega_{p}$. The Floquet-Bloch eigenmodes corresponding to the subspectrum $\sigma_{E}$ mostly reside in the region $\Sigma^{\alpha}$ filled with the optically dense component.

The intervals $\left[a_{n}, b_{n}\right]$ in (5) can be described as follows: they behave asymptotically as $\left[\widetilde{w} \theta_{n}^{-}, \widetilde{w} \theta_{n}^{+}\right]$, where

$$
\widetilde{w}=\frac{w}{1-\epsilon^{-1}}=\frac{1}{\alpha(\epsilon-1)} \sim w
$$

and the constant (i.e., not depending on the parameters of the problem) intervals $\left[\theta_{n}^{-}, \theta_{n}^{+}\right]$can be well approximated by the set

$$
[0,4] \bigcup\left\{\bigcup_{n>0}[2 \pi n, 2 \pi n+\pi]\right\}
$$

in the sense that

$$
\lim _{n \rightarrow \infty}\left|\theta_{n}^{-}-2 \pi n\right|=0, \lim _{n \rightarrow \infty}\left|\theta_{n}^{+}-2 \pi n-\pi\right|=0 .
$$

This result shows that under the conditions (1) any finite part of the spectrum $\sigma$ consists of two parts superimposed: $\sigma_{H}$ is localized around the (discrete) Dirichlet spectrum in the air bubble and is produced by the waves confined mostly to the "air bubbles"; $\sigma_{E}$ ("the fine spectrum") has the fine structure of the set $\bigcup_{n>0}\left[\theta_{n}^{-}, \theta_{n}^{+}\right]$rescaled by the small parameter $\widetilde{w}$. In particular, one can open up an arbitrary number of small gaps separated by small spectral bands in an arbitrary part of the spectrum.

One of our main aims here is to understand what operator is responsible for the spectrum $\bigcup_{n \geq 0}\left[\theta_{n}^{-}, \theta_{n}^{+}\right]$and why it is necessary to rescale this spectrum by the small parameter $\widetilde{\widetilde{w}}$. This problem will be resolved for general geometry in arbitrary dimension and under weaker asymptotic conditions than (1), namely

$$
\alpha \rightarrow 0 \text { and } w=(\epsilon \alpha)^{-1} \rightarrow W \neq \infty .
$$

Let us formulate the main result of the paper. The spectrum we are interested in can be naturally described in terms of the following operator pencil in $L^{2}\left(\mathbb{R}^{d}\right)$ :

$$
P(\theta)=-\Delta-\theta \delta_{\Sigma}-\theta W,
$$

where $\Delta$ is the Laplace operator in $\mathbb{R}^{d}, \theta$ is a scalar parameter (playing the role of the spectral parameter), $W$ is defined in (8), and $\delta_{\Sigma}$ is the $\delta$-function of the surface $\Sigma$ (i.e., the distribution that integrates test functions over $\Sigma$ ). The rigorous definition of $P(\theta)$ as a self-adjoint operator in $L^{2}\left(\mathbb{R}^{d}\right)$ requires introduction of the appropriate quadratic forms and some analysis, which are provided in Section 3. We will call the set of values $\theta$, for which the operator $P(\theta)$ is not invertible, the spectrum of the pencil $P(\theta)$ :

$$
\sigma(P)=\left\{\theta \mid P(\theta) \text { is not invertible in } L^{2}\left(\mathbb{R}^{d}\right)\right\}
$$

Let us introduce a new spectral parameter $\theta$ related to the primary spectral parameter $\lambda$ as follows:

$$
\theta=w^{-1} \lambda=(\epsilon \alpha)^{-1} \lambda
$$

The next theorem is the main result of this paper. 
THEOREM 2. Let $[a, b]$ be any finite interval of the $\theta$-axis. Then under the conditions (8) the intersection of the spectrum of the problem (3) (in terms of the spectral parameter $\theta(10))$ with the interval $[a, b]$ tends to the intersection with $[a, b]$ of the spectrum of the pencil (9). In particular, when $W=0$, i.e., when $\epsilon \alpha \rightarrow \infty$, any finite part of the spectrum of (3) tends to the corresponding part of the spectrum of the pencil $-\Delta-\theta \delta_{\Sigma}$.

A corollary on convergence of Floquet-Bloch eigenfunctions will also be proven.

Let us compare this statement with the statement of Theorem 1. In that theorem we assumed that $W=0$. If we now look at the relation to (10) we will find that after rescaling and switching to the parameter $\theta$ in the limit (1) the spectrum $\sigma_{H}$ lifts up and disappears at infinity. Hence, the spectrum of the pencil $-\Delta-\theta \delta_{\Sigma}$ is associated only with the "fine" spectrum $\sigma_{E}$. In particular, we will show that the spectrum of this pencil easily leads to the asymptotic relations (7) of the intervals $\left[\theta_{n}^{-}, \theta_{n}^{+}\right]$. This implies that the pencil $-\Delta-\theta \delta_{\Sigma}$ is the one that governs propagation of waves confined to the thin, dense component of the medium. The conclusion is that to understand the spectrum and behavior of such waves one needs to study spectral properties of this operator pencil.

Theorem 1 also provides detailed asymptotic representation for the spectrum $\sigma_{H}$, which Theorem 2 does not. The latter, on the other hand, covers any dimension and arbitrary geometry of the lattice of periods and of the "air bubbles" $\Omega_{p}$. Moreover, the case when $W \neq 0$ is now covered (it could not be included into Theorem 1 , since (1) requires that $W=0)$. Here the spectrum $\sigma_{H}$ does not go to infinity anymore and also is presented in the limit. This case of $W \neq 0$ was not covered by Theorem 1 at all. Another advantage of our current result is that it provides some new approaches to numerics, which will be discussed elsewhere. We would like to mention also that the proofs of the results of this paper are much simpler than the ones of [7], [8], and [9] (on the other hand, the results from [9] cover any finite portion of the spectrum, whereas Theorem 2 describes for $W=0$ only a finite number of the first bands).

The spectrum of the pencil $-\Delta-\theta \delta_{\Sigma}-\theta W$ can sometimes be described in terms of the spectrum of an interesting pseudodifferential operator on $\Sigma$ (a kind of the popular Dirichlet-to-Neumann map). This operator is defined as follows (the details will be described in the subsequent sections): let $\varphi$ be a function (from an appropriate functional class) on the surface $\Sigma$. Using $\varphi$ as the boundary data, we solve in each of the polygons $\Omega_{p}$ the Dirichlet boundary value problem for the equation $-\Delta u=\lambda u$. The resulting functions in different polygons match on the common boundaries, but their normal derivatives do not match. The jump of the normal derivative across $\Sigma$ gives another function $\psi$. This way we determine the Dirichlet-to-Neumann operator $\mathcal{N}(\lambda): \varphi \rightarrow \psi$. Now the spectrum of the pencil (9) can be found from the following spectral problem for the operator $\mathcal{N}(\lambda)$ :

$$
\theta \in \sigma(\mathcal{N}(\theta W)) .
$$

Hence, the original spectral problem (3) in the limit (8) reduces to a spectral problem for the (self-adjoint) operator $\mathcal{N}$. However, this reduction faces some additional complications. First of all, the spectral parameter $\theta$ enters in a nonlinear way, except when $W=0$ (in that case one gets the standard spectral problem for the operator $\mathcal{N}(0)$ ). Secondly, when $\lambda$ belongs to the spectrum of the Dirichlet Laplacian in $\Omega_{p}$, the operator $\mathcal{N}(\lambda)$ cannot be defined. Finally, rigorous definition of $\mathcal{N}$ as of a selfadjoint operator apparently requires additional conditions on the polygons. Hence, consideration of the operator pencil (9) is preferable in comparison with using the Dirichlet-to-Neumann map. 
We adopt the following notation:

$L^{2}(\Omega)$ is the Hilbert space of complex valued square integrable functions on $\Omega$.

$H^{1}(\Omega)$ is the Sobolev space of functions $u$ equipped with the norm

$$
\|u\|_{H^{1}(\Omega)}^{2}=\int_{\Omega}\left(|u(x)|^{2}+|\nabla u(x)|^{2}\right) d x .
$$

$H^{2}(\Omega)$ is the Sobolev space of functions $u$ equipped with the norm

$$
\|u\|_{H^{2}(\Omega)}^{2}=\int_{\Omega}\left(|u(x)|^{2}+|\nabla u(x)|^{2}+\sum_{i, j}\left|\partial_{i j}^{2} u(x)\right|^{2}\right) d x .
$$

2. Heuristic arguments. This section is devoted to some heuristic arguments that show how two spectra, $\sigma_{H}$ and $\sigma_{E}$, can arise. We make all these considerations rigorous in the consequent sections of this paper.

Our main object is the problem

$$
-\Delta \varphi(\mathbf{x})=\lambda \varepsilon(\mathbf{x}) \varphi(\mathbf{x}), \mathbf{x} \in \mathbb{R}^{d}
$$

under the conditions in (8). Let us recall that $\varepsilon(\mathbf{x})$ is very large closely to the surface $\Sigma$ and is equal to 1 outside of some vicinity of $\Sigma$. This leads to the natural idea of singling out of $\varepsilon(\mathbf{x})$ some function that in the limit (8) approaches the surface delta-function $\delta_{\Sigma}$ which is defined as the following distribution:

$$
\left\langle\delta_{\Sigma}, \varphi\right\rangle=\int_{\Sigma} \varphi(x) d x
$$

If we introduce

$$
\rho_{\alpha}(\mathbf{x})=((\epsilon-1) \alpha)^{-1}(\varepsilon(\mathbf{x})-1)=\left\{\begin{array}{ccc}
\alpha^{-1} & \text { in } & \Sigma^{\alpha} \\
0 & \text { in } & \Omega^{\alpha}
\end{array}\right.
$$

then the following limit holds in the distributional sense:

$$
\rho_{\alpha}(\mathbf{x}) \rightarrow \delta_{\Sigma} \text { when } \alpha \rightarrow 0 .
$$

We can now rewrite the original equation (11) in the form

$$
-\Delta \varphi(\mathbf{x})=\lambda(\epsilon-1) \alpha \rho_{\alpha}(\mathbf{x}) \varphi(\mathbf{x})+\lambda \varphi(\mathbf{x}),
$$

or

$$
-\Delta \varphi(\mathbf{x})=\theta \rho_{\alpha}(\mathbf{x}) \varphi(\mathbf{x})+\theta \widetilde{w} \varphi(\mathbf{x})
$$

where

$$
\theta=\lambda(\epsilon-1) \alpha=\lambda \widetilde{w}^{-1}, \lambda=\theta \widetilde{w} .
$$

In these new notations we obtain the equation

$$
-\Delta \varphi(\mathbf{x})=\theta \rho_{\alpha}(\mathbf{x}) \varphi(\mathbf{x})+\theta \widetilde{w} \varphi(\mathbf{x}) .
$$

If $\alpha \rightarrow 0$ and $w=(\epsilon \alpha)^{-1} \rightarrow W<\infty$, then $w$ and $\widetilde{w}$ are equivalent, i.e.,

$$
\lim w \widetilde{w}=1 .
$$


In the limit (8) our problem becomes

$$
-\Delta \varphi(\mathbf{x})=\theta \delta_{\Sigma}(\mathbf{x}) \varphi(\mathbf{x})+\theta W \varphi(\mathbf{x}) .
$$

So we can expect the result of Theorem 2 to be true.

Now our goal is to rewrite this problem in a different way (namely, using the Dirichlet-to-Neumann map). Any distributional solution of this equation in $\mathbb{R}^{d}$ is smooth in each of the "faces" $\Omega_{p}$ and is continuous through the interfaces; however, the derivatives do not match. Applying in the left-hand side the Laplace operator (in the distributional sense) to this piecewise-smooth function, one gets the following reformulation of (16):

$$
\left\{\begin{array}{c}
-\Delta \varphi(\mathbf{x})=\theta W \varphi(\mathbf{x}) \text { in each of } \Omega_{p}, \\
\text { The jump of } \left.\partial_{\nu} \varphi(\mathbf{x}) \text { across } \Sigma\right\}=\theta \varphi(\mathbf{x}) \text { on } \Sigma .
\end{array}\right.
$$

Here $\partial_{\nu} \varphi(\mathbf{x})$ is the normal to $\Sigma$ derivative of $\varphi(\mathbf{x})$.

This leads to the definition of the following version of the Dirichlet-to-Neumann map. Let us assume that $\lambda$ is not in the spectrum of the Dirichlet Laplacian on $\Omega_{p}$ and consider a reasonably well-behaved and decaying function $\varphi(\mathbf{x})$ on $\Sigma$. Consider now any "face" $\Omega_{p}$ and solve the following Dirichlet problem:

$$
-\Delta u_{p}(\mathbf{x})=\lambda u_{p}(\mathbf{x}), \mathbf{x} \in \Omega_{p} ; u_{p}(\mathbf{x})=\varphi(\mathbf{x}), \mathbf{x} \in \partial \Omega_{p} .
$$

Let $\nu_{p}$ be the outward normal vector to $\partial \Omega_{p}$ and $\partial_{p}$ be the corresponding normal derivative. The following formula defines a function on $\Sigma$ that we denote by $A_{p} \varphi$ :

$$
A_{p} \varphi(x)=\left\{\begin{array}{clc}
\partial_{p} u_{p}(x) & \text { for } & x \in \partial \Omega_{p}, \\
0 & \text { for } & x \in \Sigma-\partial \Omega_{p} .
\end{array}\right.
$$

Now we introduce the Dirichlet-to-Neumann map by the formula

$$
\mathcal{N}_{\Sigma}(\lambda) \varphi(\mathbf{x})=\sum_{p} A_{p} \varphi(x), x \in \Sigma .
$$

Notice that due to the outward directions of the normal vectors, the two normal derivatives from the opposite sides of $\Sigma$ in (18) are actually subtracted one from another. Observe now that the problem (16) can be rewritten as the eigenvalue problem for our Dirichlet-to-Neumann map:

$$
\mathcal{N}_{\Sigma}(\theta W) \varphi(\mathbf{x})=\theta \varphi(\mathbf{x}), x \in \Sigma .
$$

In particular, for $W=0$ we get

$$
\mathcal{N}_{\Sigma}(0) \varphi(\mathbf{x})=\theta \varphi(\mathbf{x}), x \in \Sigma .
$$

The apparent conclusion is that the operator $\mathcal{N}_{\Sigma}$ governs propagation of waves confined to the thin, dense component of the medium.

3. Definitions of the operators. In this section we give precise construction of the operators

$$
\begin{gathered}
S(\lambda)=-\Delta-\lambda \varepsilon(x), \\
P(\theta, \lambda)=-\Delta-\theta \delta_{\Sigma}-\lambda,
\end{gathered}
$$

and the Dirichlet-to-Neumann operator $\mathcal{N}_{\Sigma}(\lambda)$. We will use the quadratic form approach, i.e., the domains of some of these operators will not be described explicitly. 
Defining the operator $-\Delta-\lambda \varepsilon(x)$ is simple. On one hand, one can define this operator according to its formula with the Sobolev space $H^{2}\left(\mathbb{R}^{d}\right) \subset L^{2}\left(\mathbb{R}^{d}\right)$ as the domain. Using the theory of elliptic operators one can verify its self-adjointness. We choose, however, an alternative way. Namely, we define the quadratic form of this operator as

$$
s_{\lambda}[u]=\|\nabla u\|_{L^{2}\left(\mathbb{R}^{d}\right)}^{2}-\lambda \int_{L^{2}\left(\mathbb{R}^{d}\right)} \varepsilon(x)|u(x)|^{2} d x .
$$

The domain of this quadratic form is chosen to be $H^{1}\left(\mathbb{R}^{d}\right)$. This form is closed, which amounts to showing that for some large constant $C$ the sum $s_{\lambda}[u]+C\|u\|_{L^{2}\left(\mathbb{R}^{d}\right)}^{2}$ is equivalent to $\|u\|_{H^{1}\left(\mathbb{R}^{d}\right)}^{2}$, which is straightforward due to boundedness of $\varepsilon(x)$. One can also check that these two definitions of the operator $S(\lambda)$ are equivalent, although we will not use this fact (only the quadratic form approach will be used).

Let us turn now to the operator $P(\theta, \lambda)$. Since

$$
P(\theta, \lambda)=P(\theta, 0)-\lambda I,
$$

we need to define only the operator $P(\theta, 0)$ in the space $L^{2}\left(\mathbb{R}^{d}\right)$ of square integrable functions in $\mathbb{R}^{d}$. We define now a quadratic form $p[u]$ with the domain $\mathcal{D}_{P}$ consisting of all functions $u \in L^{2}\left(\mathbb{R}^{d}\right)$ such that $\left.u\right|_{\Omega_{p}} \in H^{1}\left(\Omega_{p}\right)$ for any $p$ and such that the traces of $\left.u\right|_{\Omega_{p}}$ for different values of $p$ match on common boundaries (these traces are defined, due to the standard embedding theorems (see [3] and [18])). The natural quadratic form for the operator $P(\theta)=P(\theta, 0)$ is defined by

$$
p[u]=\sum_{p \in \Gamma}\|\nabla u\|_{L^{2}\left(\Omega_{p}\right)}^{2}-\theta\left\|\left.u\right|_{\Sigma}\right\|_{L^{2}(\Sigma)}^{2}, \quad u \in \mathcal{D}_{P} .
$$

Lemma 3. The form $p[u]$ with the domain $\mathcal{D}_{P}$ is closed in $L^{2}\left(\mathbb{R}^{d}\right)$.

Proof. It is sufficient to show that for some $C$ the expressions

$$
p[u]+C\|u\|_{L_{2}\left(\mathbb{R}^{d}\right)}^{2}=\sum_{p \in \Gamma}\|\nabla u\|_{L^{2}\left(\Omega_{p}\right)}^{2}-\theta\left\|\left.u\right|_{\Sigma}\right\|_{L^{2}(\Sigma)}^{2}+C \sum_{p \in \Gamma}\|u\|_{L^{2}\left(\Omega_{p}\right)}^{2}
$$

and $\|u\|_{H^{1}\left(\mathbb{R}^{d}\right)}^{2}$ are equivalent. Rewriting $p[u]+C\|u\|_{L_{2}\left(\mathbb{R}^{d}\right)}^{2}$ we get

$$
p[u]+C\|u\|_{L_{2}\left(\mathbb{R}^{d}\right)}^{2}=\|u\|_{H^{1}\left(\mathbb{R}^{d}\right)}^{2}+(C-1)\|u\|_{L_{2}\left(\mathbb{R}^{d}\right)}^{2}-\theta\left\|\left.u\right|_{\Sigma}\right\|_{L^{2}(\Sigma)}^{2} .
$$

Now we have to show that

$$
\|u\|_{H^{1}\left(\mathbb{R}^{d}\right)}^{2}+C\|u\|_{L_{2}\left(\mathbb{R}^{d}\right)}^{2}-\theta\left\|\left.u\right|_{\Sigma}\right\|_{L^{2}(\Sigma)}^{2}
$$

is equivalent to $\|u\|_{H^{1}\left(\mathbb{R}^{d}\right)}^{2}$ when the constant $C$ is large enough. First of all, according to the embedding theorems (see for instance [18] and [3])

$$
\theta\left\|\left.u\right|_{\Sigma}\right\|_{L^{2}(\Sigma)}^{2} \leq C_{1}\|u\|_{H^{1}\left(\mathbb{R}^{d}\right)}^{2},
$$

which gives

$$
\begin{gathered}
\|u\|_{H^{1}\left(\mathbb{R}^{d}\right)}^{2}+C\|u\|_{L_{2}\left(\mathbb{R}^{d}\right)}^{2}-\theta\left\|\left.u\right|_{\Sigma}\right\|_{L^{2}(\Sigma)}^{2} \\
\leq\left(1+C_{1}\right)\|u\|_{H^{1}\left(\mathbb{R}^{d}\right)}^{2}+C\|u\|_{L_{2}\left(\mathbb{R}^{d}\right)}^{2} \leq C_{2}\|u\|_{H^{1}\left(\mathbb{R}^{d}\right)}^{2} .
\end{gathered}
$$


Now we have to show that for some constants $C_{1}, C_{2}$

$$
\|u\|_{H^{1}\left(\mathbb{R}^{d}\right)}^{2} \leq C_{1}\|u\|_{H^{1}\left(\mathbb{R}^{d}\right)}^{2}+C_{2}\|u\|_{L_{2}\left(\mathbb{R}^{d}\right)}^{2}-C_{1} \theta\left\|\left.u\right|_{\Sigma}\right\|_{L^{2}(\Sigma)}^{2},
$$

or

$$
\left\|\left.u\right|_{\Sigma}\right\|_{L^{2}(\Sigma)}^{2} \leq \frac{C_{1}-1}{C_{1} \theta}\|u\|_{H^{1}\left(\mathbb{R}^{d}\right)}^{2}+\frac{C_{2}}{C_{1} \theta}\|u\|_{L_{2}\left(\mathbb{R}^{d}\right)}^{2} .
$$

In other words, we need to show that for any $\epsilon>0$ there exists $C_{\epsilon}>0$ such that

$$
\left\|\left.u\right|_{\Sigma}\right\|_{L^{2}(\Sigma)}^{2} \leq \epsilon\|u\|_{H^{1}\left(\mathbb{R}^{d}\right)}^{2}+C_{\epsilon}\|u\|_{L_{2}\left(\mathbb{R}^{d}\right)}^{2} .
$$

Let us first consider a cylindric domain $\Omega=[0, a] \times V$. Then in the corresponding coordinates $\mathbf{x}=(x, y) \in[0, a] \times V$ the standard trace theorem says that

$$
\|u(0, y)\|_{L^{2}(V)}^{2} \leq C\|\nabla u\|_{L^{2}(\Omega)}^{2}+C\|u\|_{L^{2}(\Omega)}^{2} .
$$

Applying this inequality to the function $\widetilde{u}(\mathbf{x})=u\left(\epsilon C^{-1} x, y\right)$ and changing the variables $\epsilon C^{-1} x=x^{\prime}$ afterward we get

$$
\|u(0, y)\|_{L^{2}(V)}^{2} \leq \epsilon\|\nabla u\|_{L^{2}(\Omega)}^{2}+C_{\epsilon}\|u\|_{L^{2}(\Omega)}^{2} .
$$

If the domain becomes cylindric after a linear change of variables, the same type of estimate (with a different constant) holds. Since $\Omega_{p}$ can be covered by a finite number of such subcylinders with bases in $\partial \Omega_{p}$, one can easily conclude that the estimate (22) is true. This completes the proof of the lemma.

We will define now the Dirichlet-to-Neumann operator $\mathcal{N}_{\Sigma}(\lambda)$. Our Hilbert space will be $L^{2}(\Sigma)$, i.e., the space of measurable functions on $\Sigma$ that are square integrable with respect to the Lebesgue measure on $\Sigma$. Let a number $\lambda$ be given. Assume that $\lambda$ does not belong to the spectrum of the Dirichlet Laplacian in $\Omega_{p}$. We define the following quadratic form in $L^{2}(\Sigma)$ :

$$
n_{\lambda}[\varphi]=\int_{\mathbb{R}^{d}}|\nabla u|^{2} d x,
$$

where the function $u(x)$ in $\mathbb{R}^{d}$ is defined as follows:

$$
\left\{\begin{array}{c}
-\Delta u(x)=\lambda u(x) \text { in each } \Omega_{p}, \\
\left.u\right|_{\partial \Omega_{p}}=\left.\varphi\right|_{\partial \Omega_{p}} \text { for each } p .
\end{array}\right.
$$

In other words, the function $u(x)$ solves the Dirichlet boundary value problem for the operator $(-\Delta-\lambda)$ on each "face" $\Omega_{p}$ of $\Sigma$ with the function $\varphi$ as the boundary data. We need to describe the domain of definition of this form. The domain is the space $H^{1 / 2}(\Sigma)$ defined as follows:

$$
H^{1 / 2}(\Sigma)=\left\{\varphi(x) \text { on } \Sigma \mid \exists u \in H^{1}\left(\mathbb{R}^{d}\right) \text { such that }\left.u\right|_{\Sigma}=\varphi\right\} .
$$

The norm of $\varphi \in H^{1 / 2}(\Sigma)$ is defined as the minimum of $\|u\|_{H^{1}\left(\mathbb{R}^{d}\right)}^{2}$ over all $u$ in (24).

LEMMA 4. If $\lambda$ is not in the spectrum of the Dirichlet Laplacian in $\Omega_{p}$ and if $\Omega_{p}$ is convex, then the form $n_{\lambda}[\varphi]$ with the domain $H^{1 / 2}(\Sigma)$ is defined and closed in $L^{2}(\Sigma)$. 
Proof. We have already shown that for $\lambda$ not in the spectrum of the Dirichlet Laplacian in $\Omega_{p}$ the form is defined. It is sufficient to show now that the expressions $n_{\lambda}[\varphi]+\|\varphi\|_{L^{2}(\Sigma)}^{2}$ and $\|\varphi\|_{H^{1 / 2}(\Sigma)}^{2}$ are equivalent. Let $u(x)$ be the solution of (23) in each of $\Omega_{p}$. Then

$$
\|u\|_{H^{1}\left(\mathbb{R}^{d}\right)}^{2}=\|u\|_{L^{2}\left(\mathbb{R}^{d}\right)}^{2}+\|\nabla u\|_{L^{2}\left(\mathbb{R}^{d}\right)}^{2}=\|u\|_{L^{2}\left(\mathbb{R}^{d}\right)}^{2}+n_{\lambda}[\varphi] .
$$

Due to standard theorems on elliptic boundary value problems (see for instance [10] and [3]) applied to each "face" $\Omega_{p}$, the expressions $\|u\|_{H^{1}\left(\mathbb{R}^{d}\right)}^{2}$ and $\left\|\left.u\right|_{\Sigma}\right\|_{H^{1 / 2}(\Sigma)}^{2}=$ $\|\varphi\|_{H^{1 / 2}(\Sigma)}^{2}$ are equivalent. Using this and trace theorems we get the inequality

$$
n_{\lambda}[\varphi]+\|\varphi\|_{L^{2}(\Sigma)}^{2} \leq C\|u\|_{H^{1}\left(\mathbb{R}^{d}\right)}^{2} \leq C\|\varphi\|_{H^{1 / 2}(\Sigma)}^{2},
$$

where $C$ denotes different constants.

Now we have to show that

$$
\|\varphi\|_{H^{1 / 2}(\Sigma)}^{2} \leq C\left(n_{\lambda}[\varphi]+\|\varphi\|_{L^{2}(\Sigma)}^{2}\right) .
$$

Due to the equivalence of norms mentioned above, we conclude that

$$
\|\varphi\|_{H^{1 / 2}(\Sigma)}^{2} \leq C\|u\|_{H^{1}\left(\mathbb{R}^{d}\right)}^{2}=C\left(n_{\lambda}[\varphi]+\|u\|_{L^{2}\left(\mathbb{R}^{d}\right)}^{2}\right) .
$$

Hence, it is sufficient to show that

$$
\|u\|_{L^{2}\left(\mathbb{R}^{d}\right)}^{2} \leq C\left(n_{\lambda}[\varphi]+\|\varphi\|_{L^{2}(\Sigma)}^{2}\right) .
$$

In other words, we have to show that

$$
\|u\|_{L^{2}\left(\boldsymbol{\Omega}_{p}\right)}^{2} \leq C\left(\|\nabla u\|_{L^{2}\left(\boldsymbol{\Omega}_{p}\right)}^{2}+\|u\|_{L^{2}\left(\partial \boldsymbol{\Omega}_{p}\right)}^{2}\right)
$$

for any $p$. Let

$$
c_{p}=\frac{1}{\operatorname{Vol}\left(\Omega_{p}\right)} \int_{\Omega_{p}} u(x) d x .
$$

Then, according to the Poincaré inequality [3],

$$
\left\|u-c_{p}\right\|_{H^{1}\left(\Omega_{p}\right)} \leq C\|\nabla u\|_{L_{2}\left(\Omega_{p}\right)} .
$$

Now we have

$$
\|u\|_{H^{1}\left(\Omega_{p}\right)} \leq C\|\nabla u\|_{L_{2}\left(\Omega_{p}\right)}+\left|c_{p}\right|
$$

It remains to get an estimate

$$
\left|c_{p}\right| \leq C\left(\|\nabla u\|_{L_{2}\left(\boldsymbol{\Omega}_{p}\right)}+\|u\|_{L_{2}\left(\partial \boldsymbol{\Omega}_{p}\right)}\right),
$$

which can be justified as follows: if we have a cylindric domain $\Omega=[0, a] \times V$, then in the corresponding coordinates $\mathbf{x}=(x, y) \in[0, a] \times V$ the computation is

$$
\begin{gathered}
\left|\int_{\Omega} u(\mathbf{x}) d \mathbf{x}\right|=\left|\int_{0}^{a} \int_{V}\left(u(0, y)+\int_{0}^{x} \partial_{x} u(\tau, y) d \tau\right) d y d x\right| \\
\leq C\|u\|_{L_{2}(V)}+\int_{0}^{a} \int_{V} \int_{0}^{x}\left|\partial_{x} u(\tau, y)\right| d \tau d y d x \leq C\|u\|_{L_{2}(V)}+C\|\nabla u\|_{L_{2}(\boldsymbol{\Omega})} .
\end{gathered}
$$

If the domain becomes cylindric after a linear change of variables, the same type of estimate (with a different constant) holds. Since $\Omega_{p}$ can be covered by a finite number of such subcylinders with bases in $\partial \Omega_{p}$, the estimate (25) holds. This finishes the proof of the lemma.

Now all our self-adjoint operators are defined by their quadratic forms. 
4. Proof of the main result. In this section we provide the proof of Theorem 2 formulated in the introduction. Namely, we show that under the asymptotic relation (8) the spectrum of the operator pencil $S(\theta \widetilde{w})$ defined by $(20)$ and rescaled according to (10) tends to the spectrum of the pencil (21). Under additional conditions discussed above, the main statement also can be reformulated in terms of the Dirichlet-toNeumann operator $\mathcal{N}$. For instance, when $W=0$ we observe that after the rescaling any part of the spectrum that is not infinitesimally close to zero goes to infinity, so we are essentially discussing only the behavior of the lower part of the spectrum of (11) of order $w=(\epsilon \alpha)^{-1}$. Since the subspectrum $\sigma_{H}$ is well separated from zero, it disappears in the limit after rescaling, so we are describing the part $\sigma_{E}$ only. This explains the nature of $\sigma_{E}$ discovered in [7], [8], [9]. However, our proof now covers any periodic geometry and arbitrary dimension and the condition $W=0$ is relaxed to arbitrary values of $W<\infty$.

For the sake of simplicity we give the proof for the case of the integer lattice of periods $\Gamma=\mathbb{Z}^{d}$ only. The entire proof can be carried out without any substantial changes for general lattices. Notice that no constraints are imposed on the geometry of the polyhedral domains $\Omega_{p}$.

THEOREM 5. After introducing a new rescaled spectral parameter $\theta=\lambda \widetilde{w}^{-1}$ any finite part of the spectrum of the problem

$$
-\Delta u(\mathbf{x})=\theta \rho_{\alpha} u(\mathbf{x})+\theta \widetilde{w} u(x), \mathbf{x} \in \mathbb{R}^{d}
$$

tends (under the conditions (8)) to the spectrum of the problem

$$
-\Delta u(\mathbf{x})=\theta \delta_{\Sigma} u(\mathbf{x})+W u(x), \mathbf{x} \in \mathbb{R}^{d} .
$$

Proof of Theorem 5. The spectrum of the problem (26) will be denoted as $\sigma_{\alpha}(S)$ (although it actually depends on both $\epsilon$ and $\alpha$ ). The spectrum of (27) will be denoted as $\sigma_{\alpha}(P)$. Both problems $(26)$ and $(27)$ are periodic with respect to the lattice $\mathbb{Z}^{d}$. The standard Floquet theory arguments (see, for instance, [14], [15], and [19]) lead to the following representation for the spectra of these problems:

$$
\sigma(P)=\bigcup_{k} \sigma\left(P_{k}\right)
$$

where $\sigma\left(P_{k}\right)$ is the spectrum of the problem

$$
\left\{\begin{array}{c}
-\Delta u=\theta \delta_{\Sigma} u+W u \\
u(x+\gamma)=e^{i k \cdot \gamma} u(x) \text { for any } \gamma \in \mathbb{Z}^{d} \text { and } x \in \mathbb{R}^{d} .
\end{array}\right.
$$

The quasimomentum $k$ can be chosen in the cube (Brillouin zone)

$$
\mathcal{B}=\left\{k|| k_{j} \mid \leq \pi, j=1, \ldots, d\right\} .
$$

The same assertion is true for the spectrum of $(26)$ :

$$
\sigma_{\alpha}=\bigcup_{k} \sigma_{\alpha}(k)
$$

where $\sigma_{\alpha}(k)$ is the spectrum of the problem

$$
\left\{\begin{array}{c}
-\Delta u(\mathbf{x})=\theta \rho_{\alpha}(\mathbf{x}) u(\mathbf{x})+\theta \widetilde{w} u(\mathbf{x}), \\
u(x+\gamma)=e^{i k \cdot \gamma} u(x) \text { for any } \gamma \in \mathbb{Z}^{d} \text { and } x \in \mathbb{R}^{d} .
\end{array}\right.
$$


After commuting with the exponent $e^{i k \cdot x}$, one can reduce both problems to spectral problems on the unit torus, i.e., on periodic functions

$$
-\Delta_{k} u=\theta \delta_{\Sigma} u+W u
$$

and

$$
-\Delta_{k} u(\mathbf{x})=\theta \rho_{\alpha}(\mathbf{x}) u(\mathbf{x})+\theta \widetilde{w} u(\mathbf{x})
$$

correspondingly, where the functions $u(x)$ are periodic with respect to the lattice $\mathbb{Z}^{d}$ (and therefore can be treated as functions on the torus $\mathbb{T}^{n}=\mathbb{R}^{n} / \mathbb{Z}^{d}$ ). The differential expression $-\Delta_{k}$ is defined as

$$
-\Delta_{k}=(i \nabla-k)^{2} .
$$

The expansions (30) and (28) enable us to consider the problems (32) and (33) on a compact manifold instead of the entire space. In particular, we will prove appropriate convergence of the spectra $\sigma_{\alpha}(k)$ to $\sigma\left(P_{k}\right)$.

Distributional solutions of both problems (32) and (33) automatically belong to the Sobolev space $H^{1}\left(\mathbb{T}^{d}\right)$ (according to the definition of the operators). So, we will consider only functions $u(x)$ from this space. The expression $\left(-\Delta_{k}\right)$ determines a bounded operator from the space $H^{1}\left(\mathbb{T}^{d}\right)$ into $H^{-1}\left(\mathbb{T}^{d}\right)$. Let us denote this operator by $A(k)$ :

$$
A(k): H^{1}\left(\mathbb{T}^{d}\right) \rightarrow H^{-1}\left(\mathbb{T}^{d}\right), \quad A(k) u(x)=-\Delta_{k} u(x),
$$

where $-\Delta_{k}$ is applied in the distributional sense. $A(k)$ depends continuously (and even analytically) on the quasimomentum $k$. This follows immediately from the explicit formula

$$
A(k)=-\Delta-2 i k \cdot \nabla+k^{2} .
$$

This operator is invertible for all $k \in \mathcal{B}$ except $k=0$, which follows from the Fourier expansions

$$
u(x)=\sum_{l \in \mathbb{Z}^{d}} u_{l} e^{2 \pi i l \cdot x}, \quad A(k) u(x)=\sum_{l \in \mathbb{Z}^{d}}(2 \pi l+k)^{2} u_{l} e^{2 \pi i l \cdot x} .
$$

This "bad" point $k=0$ will cause only some minor problems later on. Note that the operator $A(0)$ is a Fredholm operator of index zero with one-dimensional kernel and cokernel (this follows from the Fourier series representation).

Consider now the following operators acting from $H^{1}\left(\mathbb{T}^{d}\right)$ into $H^{-1}\left(\mathbb{T}^{d}\right)$ :

$$
B_{\alpha}: H^{1}\left(\mathbb{T}^{d}\right) \rightarrow H^{-1}\left(\mathbb{T}^{d}\right), \quad B_{\alpha} u(x)=\rho_{\alpha}(x) u(x)+\widetilde{w} u(x)
$$

and

$$
B: H^{1}\left(\mathbb{T}^{d}\right) \rightarrow H^{-1}\left(\mathbb{T}^{d}\right), \quad B u(x)=\delta_{\Sigma}(x) u(x)+W u(x) .
$$

LEMMA 6. The operators $B_{\alpha}$ and $B$ are compact operators from $H^{1}\left(\mathbb{T}^{d}\right)$ into $H^{-1}\left(\mathbb{T}^{d}\right)$.

Proof. The operator $u(x) \rightarrow \rho_{\alpha}(x) u(x)$ factors into the composition of two operators: the first one multiplies $u(x) \in H^{1}\left(\mathbb{T}^{d}\right)$ by $\rho_{\alpha}(x)$. This is obviously a bounded operator from $H^{1}\left(\mathbb{T}^{d}\right)$ into $L^{2}\left(\mathbb{T}^{d}\right)$. The second operator is just the embedding of $L^{2}\left(\mathbb{T}^{d}\right)$ 
into $H^{-1}\left(\mathbb{T}^{d}\right)$, and it is compact due to standard embedding theorems. The embedding operator $u(x) \rightarrow \widetilde{w} u(x)$ from $H^{1}\left(\mathbb{T}^{d}\right)$ into $H^{-1}\left(\mathbb{T}^{d}\right)$ is also compact. Hence, the operator $B_{\alpha}$ is compact.

Consider the operator $B$ now. The term $W u(x)$ is proportional to the embedding of $L^{2}\left(\mathbb{T}^{d}\right)$ into $H^{-1}\left(\mathbb{T}^{d}\right)$ and, hence, is compact. The main term to discuss is $\delta_{\Sigma} u$. It also factors in the way similar to the one we applied to $B_{\alpha}$. The only difference is that multiplication by $\delta_{\Sigma}(x)$ does not act as a continuous operator from $H^{1}\left(\mathbb{T}^{d}\right)$ into $L^{2}\left(\mathbb{T}^{d}\right)$. However, it acts between $H^{1}\left(\mathbb{T}^{d}\right)$ and some $H^{-q}\left(\mathbb{T}^{d}\right)$ with $q<1$. Then the compact embedding of $H^{-q}\left(\mathbb{T}^{d}\right)$ into $H^{-1}\left(\mathbb{T}^{d}\right)$ completes the proof. So, let us find the number $q$. We claim that any $q \in\left(\frac{1}{2}, 1\right)$ is suitable. Namely, we need to show that for $u \in H^{1}\left(\mathbb{T}^{d}\right)$ the distribution $\delta_{\Sigma} u$ belongs to $H^{-q}\left(\mathbb{T}^{d}\right)$. In other words, it suffices to show that the value $\left(\delta_{\Sigma} u, v\right)$ is defined for any $u \in H^{1}\left(\mathbb{T}^{d}\right)$ and $v \in H^{q}\left(\mathbb{T}^{d}\right)$. Note that

$$
\left(\delta_{\Sigma} u, v\right)=\int_{\Sigma / \mathbb{Z}^{d}} u(x) v(x) d x .
$$

Due to standard embedding theorems, the spaces $H^{1}\left(\mathbb{T}^{d}\right)$ and $H^{q}\left(\mathbb{T}^{d}\right)$ for any $q \in$ $\left(\frac{1}{2}, 1\right)$ are continuously embedded into the space $C\left(\Sigma / \mathbb{Z}^{d}\right)$ of continuous periodic functions on the surface $\Sigma$. Hence, the expression (34) defines a continuous bilinear form on $H^{1}\left(\mathbb{T}^{d}\right) \times H^{q}\left(\mathbb{T}^{d}\right)$, which proves that $\delta_{\Sigma} u$ belongs to $H^{-q}\left(\mathbb{T}^{d}\right)$ and finishes the proof of the lemma.

Now our two spectral problems can be rewritten as

$$
A(k) u=\theta B u, \quad A(k) u=\theta B_{\alpha} u .
$$

The previous lemma implies the following important statement.

COROLLARY 7. The operators $A(k)-\theta B$ and $A(k)-\theta B_{\alpha}$ are Fredholm operators from $H^{1}\left(\mathbb{T}^{d}\right)$ into $H^{-1}\left(\mathbb{T}^{d}\right)$. The operator $A(k)-\theta B$ (respectively, $A(k)-\theta B_{\alpha}$ ) is invertible if and only if $\theta \notin \sigma\left(P_{k}\right)$ (respectively, $\theta \notin \sigma_{\alpha}(k)$ ).

Proof. Fredholmity follows from the Fredholmity of $A(k)$ and from compactness of $B$ and $B_{\alpha}$. Since $A(k)$ has index zero, the same is true for $A(k)-\theta B$ and $A(k)-\theta B_{\alpha}$. A Fredholm operator of index zero is noninvertible only if it has a nontrivial kernel, which finishes the proof of the corollary.

The next assertion is now trivial.

COROLlaRY 8. The spectra $\sigma\left(P_{k}\right)$ and $\sigma_{\alpha}(k)$ are discrete for any $k$ and depend continuously on $k$.

We also need the following statement.

LEMMA 9. Under the condition (8) we have the following convergence in the operator norm from $H^{1}\left(\mathbb{T}^{d}\right)$ into $H^{-1}\left(\mathbb{T}^{d}\right)$ :

$$
\left\|B_{\alpha}-B\right\|_{H^{1} \rightarrow H^{-1}}=0 .
$$

Proof. Since the norm of the difference operator $u \rightarrow(\widetilde{w}-W) u$ tends to zero due to the asymptotic (8), we can neglect this part. Doing this, we estimate the norm $\left\|B_{\alpha}-B\right\|_{H^{1} \rightarrow H^{-1}}$ by its definition:

$$
\begin{gathered}
\left\|B_{\alpha}-B\right\|_{H^{1} \rightarrow H^{-1}}=\sup _{u \neq 0} \frac{\left\|\left(\rho_{\alpha}(x)-\delta_{\Sigma}(x)\right) u(x)\right\|_{H^{-1}}}{\|u(x)\|_{H^{1}}} \\
=\sup _{\|u\|_{H^{1}}=\|v\|_{H^{1}}=1}\left(\left(\rho_{\alpha}-\delta_{\Sigma}\right) u, v\right) .
\end{gathered}
$$


So we have to estimate from the above expression $\left(\left(\rho_{\alpha}-\delta_{\Sigma}\right) u, v\right)$ to show that it tends to zero uniformly with respect to $\|u\|_{H^{1}}=\|v\|_{H^{1}}=1$. Polarization of this bilinear form enables us to set $u=v$ and to consider just

$$
\left(\left(\rho_{\alpha}-\delta_{\Sigma}\right) u, u\right)=\frac{1}{\alpha} \int_{\Sigma_{\alpha}}|u(x)|^{2} d x-\int_{\Sigma}|u(x)|^{2} d \sigma .
$$

Here $d \sigma$ is the surface measure on $\Sigma$ and we abuse notations identifying the sets $\Sigma$ and $\Sigma_{\alpha}$ with their images $\Sigma / \mathbb{Z}^{d}$ and $\Sigma_{\alpha} / \mathbb{Z}^{d}$ in the torus $\mathbb{T}^{d}$. Now let us assume first that we are dealing with a cylindric domain $\Omega=[0, a] \times V$ and $\mathbf{x}=(x, y) \in[0, a] \times V$ are the corresponding coordinates. Then we can write

$$
\begin{gathered}
\left(\left(\rho_{\alpha}-\delta_{\Sigma}\right) u, u\right)=\frac{1}{\alpha} \int_{V} \int_{0}^{\alpha}\left(|u(x, y)|^{2}-|u(0, y)|^{2}\right) d x d y \\
\leq \frac{1}{\alpha} \int_{V} \int_{0}^{\alpha}|u(x, y)-u(0, y)||u(x, y)+u(0, y)| d x d y \\
\leq \frac{1}{\alpha} \int_{V} \int_{0}^{\alpha}\left(\int_{0}^{\alpha}|\nabla u(\tau, y)| d \tau\right)|u(x, y)+u(0, y)| d x d y \\
\leq \frac{\alpha^{1 / 2}}{\alpha} \int_{0}^{\alpha}\left(\int_{V} \int_{0}^{\alpha}|\nabla u(\tau, y)|^{2} d x d y\right)^{1 / 2} d \tau \times\left(\int_{V} \int_{0}^{\alpha}|u(x, y)+u(0, y)|^{2} d x d y\right)^{1 / 2} .
\end{gathered}
$$

The first integral in this product can be estimated from above when $\|u\|_{H^{1}}=1$ by $C \alpha$. The second one under the same conditions is estimated by a constant due to trace theorems. The whole expression is finally estimated by $C \alpha^{1 / 2}$ and therefore tends to zero. This argument holds also if one can arrive to a cylindric domain after a linear change of variables. Now, a neighborhood of $\Sigma$ can be covered by a finite number of such skew cylinders, which finishes the proof of the lemma.

We are ready now to complete the proof of the theorem. Let $[a, b] \subset \mathbb{R} \backslash\{0\}$ belong to the complement of $\sigma(P)$. This means that $[a, b] \subset \mathbb{R} \backslash \sigma\left(P_{k}\right)$ for any $k$ from the Brillouin zone $\mathcal{B}$. Then we get an invertible family of operators

$$
A(k)-\theta \delta_{\Sigma}-\theta W: H^{1}\left(\mathbb{T}^{d}\right) \rightarrow H^{-1}\left(\mathbb{T}^{d}\right)
$$

continuously depending on parameters $(k, \theta) \in \mathcal{B} \times[a, b]$. Since $\mathcal{B} \times[a, b]$ is compact, we conclude (see, for instance, $[22]$ ) that the inverse operator family

$$
\left(A(k)-\theta \delta_{\Sigma}-\theta W\right)^{-1}: H^{-1}\left(\mathbb{T}^{d}\right) \rightarrow H^{1}\left(\mathbb{T}^{d}\right)
$$

is also continuous, and hence

$$
M=\sup _{\mathcal{B} \times[a, b]}\left\|\left(A(k)-\theta \delta_{\Sigma}\right)^{-1}\right\|_{H^{-1} \rightarrow H^{1}}<\infty .
$$

Now, according to the previous lemma we have that when $\alpha$ and $(\widetilde{w}-W)$ are small enough, the norm of the difference

$$
\begin{gathered}
\left\|\left(A(k)-\theta \rho_{\alpha}-\theta \widetilde{w}\right)-\left(A(k)-\theta \delta_{\Sigma}-\theta W\right)\right\|_{H^{1} \rightarrow H^{-1}} \\
\leq|\theta| \cdot\left(\left\|\rho_{\alpha}-\delta_{\Sigma}\right\|_{H^{1} \rightarrow H^{-1}}+|\widetilde{w}-W|\right)
\end{gathered}
$$


becomes small uniformly with respect to $(k, \theta) \in \mathcal{B} \times[a, b]$. When this norm becomes less than $M^{-1}$, the operator family $\left(A(k)-\theta \rho_{\alpha}\right)$ becomes invertible and hence the interval $[a, b]$ does not belong to the union of spectra $\sigma_{\alpha}(k)$ and therefore to the spectrum $\sigma_{\alpha}$. On the other hand, let a point $\theta \neq 0$ belong to the spectrum $\sigma(P)$. Then for any small $\beta>0$ there exists a value $k_{0} \neq 0$ of the quasi momentum such that there is a point $\theta_{0} \neq 0$ in the $\beta$-neighborhood of $\theta$ such that $\theta_{0} \in \sigma\left(P_{k_{0}}\right)$. This means that the point $\theta_{0}^{-1}$ is an eigenvalue of the compact operator $A\left(k_{0}\right)^{-1} \delta_{\Sigma}$ in $H^{1}\left(\mathbb{T}^{d}\right)$. Since the compact operators $A\left(k_{0}\right)^{-1} \rho_{\alpha}$ converge to $A\left(k_{0}\right)^{-1} \delta_{\Sigma}$ in the operator norm, we conclude that when $\alpha$ and $(\widetilde{w}-W)$ are small enough there is a point $\theta_{1} \in \sigma_{\alpha}\left(k_{0}\right) \subset \sigma_{\alpha}$ in the $2 \beta$-vicinity of $\theta_{0}$. (Let us notice that here we had to bypass the point $k=0$, at which the operator $A(k)$ is not invertible. We also notice that this operator convergence is uniform with respect to $k_{0}$ in any compact in $B$ that does not contain the point $k=0$.) This finishes the proof of Theorem 5 .

Theorem 2 is a consequence of Theorem 5 .

The end of the proof of Theorem 5 shows that in fact we have not only convergence of spectra, but also convergence of corresponding Floquet-Bloch eigenfunctions. Namely, the following assertion holds.

COROLlaRY 10. Let us assume under the conditions of Theorem 5 that $\theta_{n}(k)$ is the nth eigenvalue of the problem (29). Suppose that $\psi_{n}(k) \in H^{1}\left(\mathbf{T}^{d}\right)$ are the corresponding eigenfunctions. Let also $\mathcal{K}$ be a subcompact of the Brillouin zone $\mathcal{B}$ such that $0 \notin \mathcal{K}$. Then for any $\xi>0$ there exist $\dot{\beta}>0$ and functions $\widehat{\theta}_{n}(k) \in \mathbf{R}$ and $\widehat{\psi}_{n}(k) \in H^{1}\left(\mathbf{T}^{d}\right)(k \in \mathcal{K})$ such that $\widehat{\psi}_{n}(k)$ is an eigenfunction of the problem (31) corresponding to the eigenvalue $\widehat{\theta}_{n}(k)$,

$$
\left|\theta_{n}(k)-\widehat{\theta}_{n}(k)\right|<\xi
$$

and

$$
\left\|\psi_{n}(k)-\widehat{\psi}_{n}(k)\right\|_{H^{1}\left(\mathbf{T}^{d}\right)}<\xi
$$

when $\alpha<\beta,\left|W-(\epsilon \alpha)^{-1}\right|<\beta$, and $k \in \mathcal{K}$.

It is necessary to prove only the convergence of eigenfunctions. It follows from the ending remarks of the previous proof about the uniform on $\mathcal{K}$ convergence of compact operators $A(k)^{-1}\left(\rho_{\alpha}+\widetilde{w}\right)$ to the compact operators $A(k)^{-1}\left(\delta_{\Sigma}+W\right)$, since convergence of compact operators implies not only convergence of spectra, but also convergence of eigenfunctions.

5. The case of square $2 \mathrm{D}$ structures. Let us consider the case that was earlier treated in the papers [7], [8], and [9], namely the 2D case of the square structure, when $\Omega_{0}$ is the unit square in the plane and when (1) holds. In this case $\Sigma$ is the graph described as the set of all points in the plane $\mathbb{R}^{2}$ having at least one integer coordinate. We will show that the result of this paper leads easily to the asymptotic (7).

Consider our spectral problem for the Dirichlet-to-Neumann map with a fixed quasi momentum $k$ :

$$
-\Delta_{\mathbf{k}} u(\mathbf{x})=\theta \delta_{\Sigma}(\mathbf{x}) u(\mathbf{x}),
$$

where $\mathbf{x}=(x, y) \in[0,1] \times[0,1]$. (We remind the reader that under the condition (1) one has $W=0$.) Due to the square structure, this problem allows separation of variables. Namely, let $u(\mathbf{x})=\varphi(x) \psi(y)$, where $u(0)=\varphi(0)=\psi(0)=1$. Then we 
have

$$
\psi(y)\left(i \partial_{x}-k_{x}\right)^{2} \varphi(x)+\varphi(x)\left(i \partial_{y}-k_{y}\right)^{2} \psi(y)=\theta \varphi(x) \psi(y) \delta(y)+\theta \psi(y) \varphi(x) \delta(x)
$$

or

$$
\begin{gathered}
\psi(y)\left(i \partial_{x}-k_{x}\right)^{2} \varphi(x)-\theta \psi(y) \varphi(x) \delta(x)=-\left[\varphi(x)\left(i \partial_{y}-k_{y}\right)^{2} \psi(y)-\theta \varphi(x) \psi(y) \delta(y)\right], \\
{\left[\left(i \partial_{x}-k_{x}\right)^{2} \varphi(x)-\theta \delta(x) \varphi(x)\right] \psi(y)=-\varphi(x)\left[\left(i \partial_{y}-k_{y}\right)^{2} \psi(y)-\theta \delta(y) \psi(y)\right] .}
\end{gathered}
$$

Separating variables, we get

$$
\begin{gathered}
\left(i \partial_{x}-k_{x}\right)^{2} \varphi(x)-\theta \delta(x) \varphi(x)=\mu^{2} \varphi(x) \\
\left(i \partial_{y}-k_{y}\right)^{2} \psi(y)-\theta \delta(y) \psi(y)=-\mu^{2} \psi(y)
\end{gathered}
$$

for some $\mu>0$, or vice versa,

$$
\begin{gathered}
\left(i \partial_{x}-k_{x}\right)^{2} \varphi(x)-\theta \delta(x) \varphi(x)=-\mu^{2} \varphi(x) \\
\left(i \partial_{y}-k_{y}\right)^{2} \psi(y)-\theta \delta(y) \psi(y)=\mu^{2} \psi(y) .
\end{gathered}
$$

So we have to consider the system

$$
\begin{gathered}
\left(i \partial_{x}-k_{x}\right)^{2} \varphi(x)-\theta \delta(x) \varphi(x)=\mu^{2} \varphi(x) \\
\left(i \partial_{y}-k_{y}\right)^{2} \psi(y)-\theta \delta(y) \psi(y)=-\mu^{2} \psi(y)
\end{gathered}
$$

with 1-periodic functions $\varphi(x)$ and $\psi(y)$. This leads to the following discriminant equations (see, for instance, [2] and [19] for the general discussion of such equations, and [7] and [9] for this particular case of $\delta$-potentials):

$$
\cos \mu-\frac{\theta}{2 \mu} \sin \mu=\cos k_{x}, \quad \cosh \mu-\frac{\theta}{2 \mu} \sinh \mu=\cos k_{y},
$$

or symmetrically

$$
\cos \mu-\frac{\theta}{2 \mu} \sin \mu=\cos k_{y}, \quad \cosh \mu-\frac{\theta}{2 \mu} \sinh \mu=\cos k_{x} .
$$

These two systems lead to the same spectra, so we will use only one of them.

In other words, the spectrum (values of $\theta$ ) corresponds to solutions of the following transcendental system:

$$
\left\{\begin{array}{l}
\left|\cos \mu-\frac{\theta}{2 \mu} \sin \mu\right| \leq 1 \\
\left|\cosh \mu-\frac{\theta}{2 \mu} \sinh \mu\right| \leq 1
\end{array}\right.
$$

where $\mu>0$. One can check that solutions of this system produce a sequence of intervals $\left[\theta_{n}^{-}, \theta_{n}^{+}\right]$for $n \geq 0$ that have properties described in the introduction. Our 
main theorem shows that these are exactly the intervals found earlier with a different approach in [7], [8], and [9]. Now a simple analysis of the system (35) enables us to get the asymptotic of $\left[\theta_{n}^{-}, \theta_{n}^{+}\right]$when $n \rightarrow \infty$ that was obtained in the papers quoted above, avoiding the complicated considerations applied there. Namely, we can see from the second inequality of (35) that when $\theta \rightarrow \infty$ the auxiliary parameter $\mu$ must also tend to infinity. So, let now $\theta, \mu \rightarrow \infty$. Let us denote

$$
a=\cosh \mu-\frac{\theta}{2 \mu} \sinh \mu
$$

and assume that $|a| \leq 1$. Then

$$
\frac{\theta}{2 \mu}=\frac{a-\cosh \mu}{\sinh \mu} \rightarrow 1
$$

This leads to the conclusion that for $\mu \rightarrow \infty$ the set of $\mu$-solutions of the inequality

$$
\left|\cos \mu-\frac{\theta}{2 \mu} \sin \mu\right| \leq 1
$$

approaches the set of solutions of the inequality $|\cos \mu-\sin \mu| \leq 1$, which consists of intervals

$$
\left[n \pi,(2 n+1) \frac{\pi}{2}\right] .
$$

Taking into account the asymptotic $\frac{\theta}{2 \mu} \rightarrow 1$ we conclude that the intervals of values of $\theta$ approach

$$
[2 n \pi,(2 n+1) \pi]
$$

which is exactly the result of $[7]$ and $[8]$ about the intervals $\left[\theta_{n}^{-}, \theta_{n}^{+}\right]$. Numerical experiments show that the convergence is very fast.

Acknowledgment. The second author expresses his gratitude to Dr. I. Ponomaryov for useful comments.

\section{REFERENCES}

[1] Development and applications of materials exhibiting photonic band gaps, J. Opt. Soc. Amer. B, 10 (1993), pp. 280-413.

[2] M. S. P. Eastham, The Spectral Theory of Periodic Differential Equations, Scottish Acad. Press, Edinburgh, London, 1973.

[3] D. E. Edmunds And W. Evans, Spectral Theory and Differential Operators, Oxford Science Publ., Clarendon Press, Oxford, 1990.

[4] A. Figotin, Photonic pseudogaps in periodic dielectric structures, J. Statist. Phys., 74 (1994), pp. 443-446.

[5] A. Figotin And P. Kuchment, Band-gap structure of the spectrum of periodic Maxwell operators, J. Statist. Phys., 74 (1994), pp. 447-458.

[6] A. Figotin And P. Kuchment, Band-gap structure of the spectrum of periodic dielectric and acoustic media, I: Scalar model, SIAM J. Appl. Math., 56 (1996), pp. 68-88.

[7] A. Figotin And P. Kuchment, Band-gap structure of the spectrum of periodic dielectric and acoustic media, II: $2 D$ photonic crystals, preprint, 1995.

[8] A. Figotin And P. Kuchment, $2 D$ photonic crystals with cubic structure: Asymptotic analysis (a shorter version of [7]), in IMA Proceedings, Vol. 96, Wave Propagation in Complex Media, G. Papanicolaou, ed., Springer-Verlag, New York, pp. 23-30, 1997. 
[9] A. Figotin And P. Kuchment, Band-gap structure of the spectrum of periodic dielectric and acoustic media, II: 2D photonic crystals, SIAM J. Appl. Math., 56 (1996), pp. 1561-1620.

[10] P. Grisvard, Elliptic Problems in Nonsmooth Domains, Pitman, Boston, MA, 1985.

[11] J. D. Joannopoulos, R. D. Meade, And J. N. Winn, Photonic Crystals: Molding the Flow of Light, Princeton University Press, Princeton, NJ, 1995.

[12] S. John, Localization of light, Phys. Today, (May 1991), pp. 32-40.

[13] S. John, The localization of light, in Photonic Band Gaps and Localization, NATO Adv. Sci. Inst. Ser. B Phys. 308, Plenum, New York, 1993.

[14] P. Kuchment, Floquet Theory for partial differential equations, Russian Math. Surveys, 37 (1982), pp. 1-60.

[15] P. Kuchment, Floquet Theory for Partial Differential Equations, Birkhäuser-Verlag, Basel, 1993.

[16] J. Maynard, Acoustic Anderson localization, in Random Media and Composites, SIAM, Philadelphia, PA, 1988.

[17] P. M. Morse and K. U. Ingard, Theoretical Acoustics, Princeton University Press, Princeton, NJ, 1986.

[18] S. M. Nikol'Skir, Approximation of Functions of Several Variables and Imbedding Theorems, Springer-Verlag, New York, 1975

[19] M. Reed And B. Simon, Methods of Modern Mathematical Physics, Vol. IV, Analysis of Operators, Academic Press, New York, 1978.

[20] P. Sheng, Scattering and Localization of Classical Waves, World Scientific, River Edge, NJ, 1990.

[21] P. R. Villeneuve And M. Piché, Photonic band gaps of transverse-electric models in twodimensionally periodic media, J. Opt. Soc. Amer. A, 8 (1991), pp. 1296-1305.

[22] M. Zaidenberg, S. Krein, P. Kuchment, And A. Pankov, Banach bundles and linear operators, Russian Math. Surveys, 30 (1975), pp. 115-175. 